\title{
Collective Conversational Peer Review of Journal Submission: A Tool to Integrate Medical Education and Practice
}

\author{
Vivek Podder ${ }^{a}$ Amy Price ${ }^{b, c}$ Madhava Sai Sivapuram ${ }^{d}$ Ashwini Ronghe ${ }^{e}$ \\ Srija Katta ${ }^{f}$ Avinash Kumar Gupta ${ }^{g}$ Rakesh Biswas ${ }^{\text {h }}$

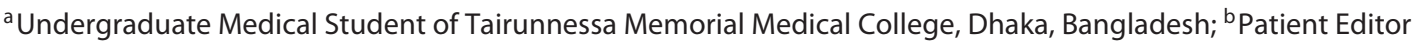 \\ (Research and Evaluation), The BMJ, London, UK; ' Continuing Education, The University of Oxford, Oxford, UK; \\ dUndergraduate Medical Student of Dr. Pinnamaneni Siddhartha Institute of Medical Sciences and Research \\ Foundation, Vijayawada, India; eUndergraduate Medical Student of Grant Medical College and Sir JJ Group of \\ Hospitals, Mumbai, India; fUndergraduate Pharmacy Student of St. Peters Institute of Pharmaceutical Sciences, \\ Warangal, India; 9 Undergraduate Medical Student of Universal College of Medical Sciences, Bhairahawa, Nepal; \\ hProfessor of Medicine, Kamineni Institute of Medical Sciences, Nalgonda, India
}

\section{Keywords}

Collective peer review · Prepublication · Conversational learning $\cdot$ Medical education · Practice

\begin{abstract}
Background: In this study, we demonstrate a collective collaborative, conversational, pre-publication peer review of a randomized controlled trial. Methods: Using Critical Appraisal Skills Programme checklist, a group of research-oriented undergraduate medical and pharmacy students and their teacher collectively on an online forum, discuss and review a randomized controlled trial submitted to the Annals of Neurosciences and the explanatory commentary from each reviewer makes a basic scaffold for critical appraisal of the manuscript. Results: This method provided the opportunity for students to engage in online interactive training and
\end{abstract}

(C) 2018 S. Karger AG, Basel

\section{E-Mail karger@karger.com \\ www.karger.com/aon \\ KARGER}

allowed them to understand tools used for critical appraisal of a study. Students were incentivized by the potential publication credit and they look forward to continuing this work and perhaps providing one small step to making medical education more interactive. Conclusion: Open peer review involving a group of reviewers at a time produces multidirectional reviewing concepts, thus helps to improve the quality of paper and also may reduce the time between review and publication.

(c) 2018 S. Karger AG, Basel

\section{Introduction}

Current undergraduate medical education systems are largely confined to rote textbook-based approaches, which lack critical appraisal training for undergraduate 
students. Basic skills for critical appraisal of available evidence are necessary for accurate clinical decisions in practice. Time constraints, lack of mentorship, and failure to establish group work in like-minded cohorts can diminish the effect of evidence-based practice from medical education. Utilizing the power of web 2.0 via social networking sites like WhatsApp, Facebook, and focal discussion groups, conversational peer review of journal submission through asynchronous conversational discussion may position medical students to make the best evidence decisions in education and in practice [1].

Peer review has long been an essential method for quality control and validation of scientific studies in medical research. In 1731, the Royal Society of Edinburgh first peer reviewed publications of medical essays and observations [2]. Peer review is used to validate the manuscripts submitted to scientific journals in order to detect major and minor flaws, to find competence, significance, and originality so that good quality papers are accepted and unreliable data are prevented from publication, and thus good quality peer review can influence patient care and accurately inform healthcare providers and policymakers $[3,4]$. Although, transparency of a peer review process allows all to learn from the peer review, the current trend of mainstream peer review is single peer reviewer and editor with minimal transparency where the review takes place behind closed curtains and authors are expected to comply and resend the recommended changes $[2,5]$. The blind review process is criticized by some as unreliable, inconsistent, contributing to costly delay, lack of accountability and risks of subversion, social and publication biases, and wastefulness [6]. This traditional peer review method could possibly be reformed by making it an open process [7].

The digital revolution sets up a stage for developing a web-based digital platform wherein a group of students can asynchronously learn through conversations even from their remote locations. In conversational learning, students and a facilitator can be actively engaged in the group discussion and through an exploratory approach can dissect each case-based problem for an evidencebased solution, creating secondary medical education resources to stimulate the next generation of medical professionals and promote clinical problem solving skills [8]. The use of creative technology and conversational learning through focus group discussion on a web-based platform, like WhatsApp, has the potential to make the traditional peer review method a collective process and to improve students' critical appraisal skills [9].

Collective Conversational Peer Review
In this study, we demonstrate a collective collaborative, conversational, pre-publication peer review of a randomized controlled trial (RCT). We aim to lift currently closed curtains of traditional peer review and illustrate a conversational, collaborative collective open peer reviewing process using the CASP checklist. Using Critical Appraisal Skills Programme (CASP) checklist [10], a group of research-oriented undergraduate medical and pharmacy students center their discussion around an RCT on a web-based forum, and the explanatory commentary from each reviewer makes a basic scaffold for understanding open the peer review process and for critical appraisal of the evidence through conversational learning. Price et al. [11] have previously used this method to train research volunteers in critical appraisal for individual studies and systematic reviews.

Most of the conversational learning published in the past is centered around patients to help in learning the best available evidence for management, but to the best of our knowledge this study will be the first one centered around an RCT.

\section{Materials and Methods}

This pilot feasibility educational methods study was planned to critically appraise and review a pre-publication randomized control trial study submitted to a journal for peer review. The prepublication randomized control trial was selected randomly from the set of manuscripts received by the editor of the journal, who acted as the moderator of our study after receiving approval from the authors and the journal.

The mode of communication for the web-based conversational learning and review of the manuscript was a WhatsApp group created by the moderator, which was encrypted and accessible only to the students who were participating in the study. The students were invited for appraisal and review of this manuscript randomly. The identities of authors of the paper and their institutions were concealed.

The manuscript was appraised using the CASP RCT checklist consisting of 11 questions (version 2017; Table 1) by 5 medical and pharmacy undergraduate students across south Asian countries [10]. During the appraisal, 3 broad issues were kept into consideration as per CASP: "are the results of the trial valid?" "what are the results?", "will the results help locally?" (Table 1). After a series of asynchronous conversations among the students enabled by the moderator, the review with positive and negative comments was agreed by the students and sent to the authors of the paper submitted for peer review. The moderator invited the authors to ask for clarifications before publishing.

\section{Data Analysis}

To preserve the originality of conversational data, it is presented in the same sequence as it happened online. The moderator is indicated by the title "moderator" and/or initials (R.B.) and student reviewers are indicated by their initials. 
Table 1. Showing CASP randomized controlled trial checklist and student responses

Question Response Comment

1. Did the trial address a clearly focused issue?

2. Was the assignment of patients

to treatments randomized?
Yes

Yes

SK: block randomization was

used in this and the randomization was concealed

AR: it was done via block randomization

AG: it was randomized but no information is given, if randomization was done computationally or not

VP: they were allocated into "experimental group," that is, pelvic stability and control group, that is, standard physiotherapy. Randomization was done through block randomization method with concealed allocation. "Block randomization works by randomizing participants within blocks such that an equal number are assigned to each treatment. A disadvantage of block randomization is that the allocation of participants may be predictable and result in selection bias when the study groups are unmasked" [12]

3. Were all of the patients who SK: yes AR: yes AG: losses to follow-up is $+23.00 \%$, but there entered the trial properly accounted for at its conclusion?

4. Were patients, health workers, and study personnel "blind" to treatment?

SK: yes
is no reason mentioned for the losses

SK: patients and the assessor were unaware of the intervention and randomization

AR: yes AR: the outcomes were collected by someone not involved in the study or randomization. Clinician was blind. For patients, it is not mentioned. Results: Can't calculate RR or NNH as no data is given. $\mathrm{P}$ value was below 0.05 so the result is statistically significant. May or may not be clinically significant.

AG: no AG: no info about blinding, either clinicians or patients were blinded or not

5. Were the groups similar at the start of the trial?

AR: no

AR: patients similar in terms of disease.

No information given

about age, sex. Can act as confounding factors

AG: yes AG: both groups were similar

VP: can't tell VP: we may need to look at Table 1, and apart from that results show, all demo variable and outcome variables were similar except $*$ FMA-LE $(p=0.008)$ and TIS $2.0(p=0.001)$

6. Aside from the experimental intervention, were the groups treated equally?
SK: can't tell SK: unclear apart from the intervention no information about additional treatment was mentioned

AR: can't tell AR: no information about additional testing and treatment given

AG: yes

SK: can't tell
AG: similar physiotherapy were given but no mention of similar other treatments, if given

\begin{tabular}{lll}
\hline 7. How large was the treatment effect? & SK: can't tell & SK: relative risk was not mentioned \\
\hline
\end{tabular}


Table 1. (continued)

\begin{tabular}{lll}
\hline Question & Response & Comment \\
$\begin{array}{l}\text { 8. How precise was the estimate } \\
\text { of the treatment effect? }\end{array}$ & RB: can't tell & $\begin{array}{l}\text { RB: looking at the WIDE confidence intervals } \\
\text { provided in their study it may not inspire much } \\
\text { confidence in their study. If only their confidence } \\
\text { intervals were narrower, it may have indicated } \\
\text { a better precision of the outcome measures }\end{array}$ \\
$\begin{array}{lll}\text { 9. Can the results be applied in } \\
\text { your context? (or to the local population?) }\end{array}$ & VP: can't tell & $\begin{array}{l}\text { VP: the sample size was small } \\
\text { to conclude generalized effectiveness of one } \\
\text { intervention over another }\end{array}$ \\
$\begin{array}{l}\text { 10. Were all clinically important } \\
\text { outcomes considered? }\end{array}$ & VP: can't tell \\
$\begin{array}{l}\text { 11. Are the benefits worth } \\
\text { the harms and costs? }\end{array}$ & VP: can't tell \\
\hline
\end{tabular}

\section{Results}

What follows below is an original version of the WhatsApp conversational review that began with an invitation statement from the moderator and each participant critically appraised and reviewed the RCT to determine its efficacy (Table 1). As the discussion progressed, dialogue between the moderator and students centered around the trial, and a review report was summarized. It is followed by the authors response to the queries asked in the review report.

$R B$ (Moderator): Can I give you an RCT report originating in India and submitted to our journal to review and discuss? I would need you all to critically appraise it using the CASP checklist for evaluating an RCT and to provide your evidence-based Medicine inputs. The PDF is confidential and not to be shared outside this group. We can continue to asynchronously discuss it until tonight. You will have to focus on how the authors determined efficacy.

\section{The Student and Moderator Discussion Content}

$A G$ : How we can decide here whether it is single or double blind?

$V P$ : Blinding is of 3 types: single blind, when the patient is blind; double blind, when the patient and the investigator are blind; and triple blind, when the patient, investigator, and data clean-up people are blind.

$R B$ : What are the different ways to practice allocation concealment? Is allocation concealment different from blinding?

Collective Conversational Peer Review
$V P$ : It is different from blinding as blinding is concerned with blinding of all 3 (investigator, patient, data analyzer) in giving interventions, whereas allocation concealment is concerned with preventing selection bias of allocating participants into different group, that is, which person will go to experimental group and which person will go to control group [13].

$R B$ : That is allocation, but how is allocation concealment done and how have these study authors done it?

$V P$ : Allocation concealment involves developing allocation concealment mechanism (such as numbered, identical bottles or sequentially numbered, sealed, opaque envelopes) and preparing the allocation concealment mechanism using the allocation sequence from the sequence generation step. They did not detail how they concealed allocation [14].

$A R$ : There are several biases involved in the study as well. One would be that only hospital patients are enrolled, that is, Berksonian bias, and may not be a representative of the general population. What were the criteria for post-stroke duration being 219 days? It is not answered. Ascertainment bias is when only hospitalized patients are taken, and they are the ones who have severe disease. Will this work in less severe cases? Selection bias considers the data from the patients who dropped out being taken or not? All these are actually theoretical and a study where there is no bias is not possible. They mentioned a couple of limitations and the implications as well. They did mention that gender ratio is skewed.

SK and AR: Details about grouping of patients with probable risk factors for stroke such as diabetes mellitus, 
hypertension, and atherosclerosis would have been better and other co-morbid conditions will have more clear idea about the patients. Analysis of demographics would have been done. Social factors like social history and alcohol consumption will add up.

$R B$ : Can any of you discuss how to detect if there were any significant differences in baseline patient characteristics in this paper? We have already had a good discussion on the patient $(\mathrm{P})$ as well as how the intervention (I) was done along with the characteristics of the comparator $(\mathrm{C})$ group and now we need to focus on the outcomes (O). Please let me know your thoughts on how the outcomes and effect size were evaluated in this paper.

$V P$ : In the baseline patient characteristics, $p$ value was $<0.05$ for gender (male/female; $p=0.006$ ), ischemic/hemorrhagic stroke ( $p=0.002)$, side affected $(p=0.016)$, FuglMeyer assessment of lower limbs $(p=0.003)$, and trunk impairment scale $2.0(p=0.00)$ that suggests there is statistically significant differences. Should it be considered post intervention only?

Could the difference be for the beneficial effects of the intervention, that is, would this difference help to assess the benefits in outcome?

$R B$ : Yes, the outcome benefits could be false due to these differences. So, let us look at the outcomes now and see if that is what happened?

$V P$ : Now treatment effect is not discussed adequately. Need to understand how to interpret it.

$A R$ : I too do not understand the effect size concept.

$R B$ : Alright, so in simple terms, it means a quantitative estimate of how beneficial (efficacious with an effect) an intervention is over the other in our patient and it tells us the quantitative benefit of pelvic stability training over standard physiotherapy. Now you would like to know how our authors calculated it. Look at Table 3. Notice the post pre-intervention change in mean values in both the pelvic stability training as well as standard physiotherapy groups. Using our common sense, how may we proceed to calculate how much pelvic stability training is better than standard physiotherapy?

$V P$ : By subtracting post pre-intervention change in standard physiotherapy group from the pelvic stability group?

$R B$ : Now when you look at those mean values and just end up subtracting them alone what would happen to the values that deviate from the mean? How would you account for them? Any other school math trick you can think of?
$V P$ : Is Table 3 a derivative of the Table 2 calculation? With information of confidence interval which can help us to know how it matches to general population!!

$R B$ : How would you take it into account? Hint: in the numerator or denominator?

$A R$ : Standard error of mean? SD/square root of sample size?

$R B$ : Just think for now where would you like to put the SD if you want to take it into account. Multiply it with the mean difference or divide the mean difference with it? OK! To break the suspense, a $\mathrm{PhD}$ psychology guy from New York figured that just dividing the mean values with the standard deviation would be good way to have an effect size and due to some strange reason he named it " $D$ " and put his name in front of it.

$V P$ : Cohen's D!

$R B$ : How do you calculate the pooled standard deviation to place it in the denominator?

VP: Root over to SD1 (Group 1) + SD2 (Group 2) over 2

$R B$ : And we may find all the necessary values in Table 2 . Now if you read a bit more on Cohen's D you may realize that these authors should have done something more to rectify their choice of Cohen's D to calculate the sample size. Here's the catch: "Cohen's D works best for larger sample sizes $(>50)$. For smaller sample sizes, it tends to over-inflate results" [15].

VP: As Cohen described Rule of Thumb Interpretation: "If we are not familiar with the meaning of standard deviations and z-scores, or have trouble visualizing what the result of Cohen's D means, use these general "rule of thumb" guidelines (which Cohen said should be used cautiously)": small effect $=0.2$, medium effect $=0.5$, large effect $=0.8$. In this study, it was 0.10 , 0.30 , and 0.50 , respectively, and effect size for all the outcome measures were large except Cadence, lateral pelvic tilt.

$R B$ : Anywhere in this article, is it mentioned if these authors used the "correction factor available to reduce effect sizes for small samples by a few percentage points?"

$A G$ : Not mentioned about correlation factor.

$V P$ : What about confidence interval? How would you match it to the population?

$R B$ : Good question. If you look at Table 3 can you tell how these CIs for each outcome measure were calculated? Looking at the WIDE CIs provided in their study it may not inspire much confidence in their study. If only their CIs were narrower it may have indicated a better precision of the outcome measures?

$R B$ : Let's make a list of the good and bad in this paper. 


\section{Review Report}

\section{Positive Comments}

1. The inclusion criteria with the patients in regard to medical condition of the patient were clearly mentioned.

2. Usage of "block randomization" appropriate for small sample sizes.

3. The study results positively matched with the purpose of the study, that is, pelvic stabilitytraining being effective over standard physiotherapy.

4. They mentioned few limitations like gender and the parameter.

Author response: many thanks for your effort in reviewing our paper and your remark on study methodology and its treatment importance on mobility and function post stroke.

\section{Negative Comments}

(1) Selection criteria: the reason for considering poststroke duration as 219 (156) days was not mentioned.

Author response: many thanks for raising this valuable point. The main reasons for considering post-stroke duration of 219 (156) days are that of possible neural reorganization in individuals with late subacute and chronic stage (within 1 year post-stroke duration) and also not showing much of permanent musculoskeletal adaptations. The pelvic stability is not only controlled by pyramidal system, but also influenced by extrapyramidal system. We hypothesized that the proximal dynamic pelvic stability influences the distal limb mobility that could possibly be retrained before the disuse/inactivity-related movement patterns and soft tissue adaptations set in. From the physical rehabilitation point of view, the pelvic treatment strategies in chronic stroke beyond 1-year post-stroke duration requires different exercise strategies including soft tissue mobilization and greater exercise intensity, that is, minimum 6-8 weeks duration. In previous trunk trials, this hypothesis has been tested for!! Sir/madam, we truly consider your valuable suggestion which gives us an idea of comparing the pelvic stability training regime and its genuine changes in acute care or chronic stage stroke in future studies.

(2) Classification of patients with respect to their risk factor for stroke was not done.

Author response: we looked into our results in descriptive table and observed that the risk factors are not quoted. Thanks for mentioning it. As we included only 34 individuals in this study, we were afraid that classification was not possible. But we rather attempted to identify the risk factors from the screening sheets and report them, that is, numbers and percentages in results section.

(3) Grouping of patients in regard to other comorbidities was not mentioned.

Author response: we agree to your point. However, we could not group the individuals post stroke based on their comorbidities such as diabetes mellitus, heart disease, hypertension, etc., as our study sample size was relatively low. As a physical rehabilitation perspective, the primary objective is to look upon the movement behavior post stroke irrespective of the risk factor comorbidities. We admit that this is a potential limitation of the study. Nevertheless, we excluded the patients with orthopedic-related musculoskeletal comorbidities from the study that possibly affect the balance and walking. In this way, both groups were comparable and similar at baseline.

(4) Social history of the patients like alcohol and tobacco consumption was not mentioned.

Author response: thanks for your suggestions. We incorporated your recommendations in results section.

(5) The way by which allocation was concealed to the patients was not mentioned.

Author response: we did mention the concealed allocation in the text, but forgot to write the way it was done. The concealed allocation was done by opaque sealed envelopes through block randomization method by an independent observer who neither participated in collecting outcome measures nor conducted the interventions.

(6) The details about the similarity of groups with respect to other treatments (excluding the intervention) were not given.

Author response: appreciate you much for addressing similarity of groups which was not mentioned in the paper earlier. All the study participants had only involved in exercise regime allocated to the group and followed a medical advice as prescribed by neurologist. None of them underwent treatments such as Ayurveda, and massage which might potentially affect the outcome of the pelvic training regime. The exercise intensity to both groups was similar during the study period.

(7) More information regarding the blinding of the trail is required.

Author response: it was an assessor blinded clinical trial. The assessor was not involved in treating the patients and allocating them into groups. In physical rehabilitation trails, both the therapist and patient were aware about the exercise regimes administered, hence, they were not blinded.

(8) The authors should have done something more to rectify their choice of Cohen's D to calculate the sam- 
ple size (Cohen's D works best for larger sample sizes $(>50)$. For smaller sample sizes, it tends to over-inflate results).

Author response: thank you for your statistic information on Cohen's D. We sought out the statistician opinion and suggested the same. We reanalyzed our data carefully and removed "D" from results section and Table 3 as well. Instead, we have rewritten the results section adding the percentage of improvement and the ratio of relative risk post intervention. The Tables 2 and 3 are merged together into single Table 2. In addition, we reported 95\% CI instead of standard deviation under within-group change between post and pre-training.

(9) Relative risk was not mentioned.

Author response: As you suggested it is incorporated in the results and discussion sections. Though, the occurrence of event cannot be reported in rehabilitation studies as in medical research, we calculated the percentage of improvement in each outcome measures from their preintervention score and then compared the ratio between the 2 groups, thus commenting on how much improvement the pelvic training groups attained over standard physiotherapy.

(10) More limitations considering bias should have been mentioned.

Author response: The following points pertain to limitations included in the text. Grouping of patients in regard to other comorbidities was not performed due to small sample size. Another potential limitation is that the strength of lower trunk muscles was not tested in this study. Nevertheless, the strength of lower limb muscles was not tested in functional position, the isokinetic strength testing in future trial might give genuine effects of pelvic stability training. We are cautioned to generalize the benefits of pelvic stability training while interpreting the findings due to following reasons. At the beginning of intervention, all the patients recruited in the study had a history of single stroke onset of supratentorial lesion, but not cerebellar or brainstem stroke lesion. They had an independent ambulating capacity with or without using a walking device. The study was conducted in single rehabilitation setting and participants were not in the acute stage of stroke recovery. We believe that following them up with more study duration would further affirm the motor learning effects of pelvic stability training in patients with stroke. We further recommend to examine the benefits of pelvic stability training on balance confidence and community participants in future clinical trials involving more number of study participants with followup.
(11) The sample size was small to conclude generalized effectiveness of one intervention over another.

Author response: we consider your suggestion. We use terminology "pelvic stability training benefits" so that we do not extrapolate the conclusion. We are cautioned to state in limitations and future recommendations that as the study was conducted in single rehabilitation setting and participants were not in the acute stage of stroke recovery, we cannot generalize the findings.

After the successful review and revision of the RCT, the paper has been published [16].

\section{Implications of the Study}

This method provides the opportunity for students to engage in online interactive training which allows them to understand the line of questioning and the tools used for critical appraisal of a study. Critical thinking and appraisal can present a steep learning curve for students and the group contribution provides the opportunity to learn these skills with others and to improve the way a learned can communicate their arguments. This experience can provide a strategy to integrate medical education with the practice of medicine. The students communicated that they learned through this experience and all were pleased that they contributed value for the study authors. In this instance, students were incentivized by the potential publication credit and they look forward to continuing this work and perhaps providing one small step to making medical education more interactive. In our study, the quality of peer review has not been compared. So, further studies are being planned to explain the quality of peer review though online conversational learning.

\section{Conclusions}

Online conversational learning facilitated and supported through use of information and communication technology aids in global connectivity. Open peer review involving a group of reviewers at a time produces multidirectional reviewing concepts, thus helps to improve the quality of paper and also may reduce the time between review and publication. This strategy creates a medium for early career researchers, students, and patient reviewers to understand the peer review process and practice the appraisal of a study for a broader multi-disciplinary perspective and the clinical application of best evidence. 


\section{Acknowledgments}

We acknowledge authors of the RCT for their useful responses and Annals of Neurosciences for providing the opportunity.

\section{Ethics Approval and Consent to Participate}

Informed consent was attained for the content for analysis from the Editor in chief of Annals of Neurosciences, Prof. Akshay Anand.

\section{Disclosure Statement}

R.B. and A.P. are consultants of the BMJ elective initiative and have collaborated to build the collaborative learning platform and materials used in this study. A.P. is the patient editor (Research and Evaluation) at The BMJ. All authors support the call for interactive medical education through conversational learning.

\section{Consent for Publication}

All contributors meet the ICMJE criteria for authorship. All authors have agreed the final version and consent to publication of the study.

\section{Data Sharing}

All data is in the publication other than the study which must remain de-identified until it is accepted for publication. Any further requests for information can be made by contacting Prof. R.B., the corresponding author.

\section{Funding Source}

This research received no specific grant from any funding agency in the public, commercial or not-for-profit sectors.

\section{Authors' Contributions}

R.B. and A.P. designed the study. V.P. and M.S.S. wrote the first draft of the manuscript with A.P. All authors contributed to the interpretation of the results and the writing of the manuscript for publication.

\section{References}

1 Bera K, Seth B, Biswas R: Conversational learning among medical students: harnessing the power of web 2.0 through user driven healthcare. Ann Neurosci 2013;20:37-38.

2 Steel G, Price A, Seth B, Biswas R, Chatterjee $\mathrm{P}$ : Charity is welcome: the international benefits and pitfalls of peer Review. PeerJ PrePrints 2016;4:e1585v2.

3 Manchikanti L, Kaye AD, Boswell MV, Hirsch JA: Medical journal peer review: process and bias. Pain Physician 2015;18:E1-E14.

4 Vercellini P, Buggio L, Viganò P, Somigliana E: Peer review in medical journals: beyond quality of reports towards transparency and public scrutiny of the process. Eur J Intern Med 2016;31:15-19.

5 Wicherts JM: Peer review quality and transparency of the peer-review process in open access and subscription journals. PLoS One 2016;11:e0147913.

6 Ross-Hellauer T: What is open peer review? A systematic review. Version 2. F1000Res 2017; 6:588.
7 Thörn A: Peer review: a closed system in need of reform. Lakartidningen 2002;99:3106-3108.

8 Biswas T, Bera K, Biswas R: Creating secondary learning resources from the BMJ Case Reports through social-media based discussion groups. BMJ Case Reports Blog 2017. http://blogs.bmj. com/case-reports/2011/10/28/creating-secondary-learning-resources.

9 Shaffer K, Small JE: Blended learning in medical education: use of an integrated approach with web-based small group modules and didactic instruction for teaching radiologic anatomy. Acad Radiol 2004;11:1059-1070.

10 Critical Appraisal Skills Programme (CASP). Critical Appraisal Skills Programme (CASP). 2017. http://www.casp-uk.net/casptools-checklists (cited November 27, 2017).

11 Price A, Albarqouni L, Kirkpatrick J, Clarke M, Liew SM, Roberts N, et al: Patient and public involvement in the design of clinical trials: An overview of systematic reviews. J Eval Clin Pract 2017, Epub ahead of print.
12 Efird J: Blocked randomization with randomly selected block sizes. Int J Environ Res Public Health 2011;8:15-20.

13 Misra S: Randomized double blind placebo control studies, the "Gold Standard" in intervention based studies. Indian J Sex Transm Dis 2012;33:131-134.

14 Moher D, Hopewell S, Schulz KF, Montori V, Gøtzsche PC, Devereaux PJ, et al: CONSORT 2010 explanation and elaboration: updated guidelines for reporting parallel group randomised trials. BMJ 2010;340: c869.

15 Cohen's D: Definition, Examples, Formulas. Statistics How to 2017. http://www.statisticshowto.com/cohens-d/ (cited November 27, 2017).

16 Dubey L, Karthikbabu S, Mohan D: Effects of pelvic stability training on movement control, hip muscles strength, walking speed and daily activities after stroke: a randomized controlled trial. Ann Neurosci 2018;25: 80-89. 